\title{
An ongoing large outbreak of measles in Merseyside, England, January to June 2012
}

R Vivancos (roberto.vivancos@hpa.org.uk) ${ }^{1}$, A Keenan 1 , S Farmer , J Atkinson $^{1}$, E Coffey , E Dardamissis $^{1}$, J Dillon ${ }^{2}$, R J Drew 3 ,

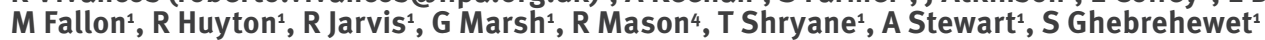

1. Cheshire \& Merseyside Health Protection Unit, Health Protection Agency, Rail House, Lord Nelson Street, Liverpool, United Kingdom

2. Liverpool Primary Care Trust, Art House, Liverpool, United Kingdom

3. Alder Hey Children's Hospital, Liverpool, United Kingdom

4. NHS Halton \& St Helens, Health Care Resource Centre, Oaks Place, Widnes, United Kingdom

Citation style for this article:

Vivancos R, Keenan A, Farmer S, Atkinson J, Coffey E, Dardamissis E, Dillon J, Drew RJ, Fallon M, Huyton R, Jarvis R, Marsh G, Mason R, Shryane T, Stewart A,

Ghebrehewet S. An ongoing large outbreak of measles in Merseyside, England, January to June 2012. Euro Surveill. 2012;17(29):pii=20226. Available online: http:// www.eurosurveillance.org/ViewArticle.aspx?Articleld=20226

Article submitted on 13 July 2012 / published on 19 July 2012

From 1 January to 30 June 2012, 359 confirmed and 157 probable cases of measles were reported in Merseyside, England. The most affected age groups were children under five years and young adults from 15 years of age. Most cases have been sporadic. There have been few outbreaks in nurseries; however, no outbreaks have been reported in schools. Of the cases eligible for vaccination, only $3 \%$ of the confirmed cases were fully immunised.

From 1 January until 30 June 2012, 1,339 suspected cases of measles were reported by clinicians (family doctors or hospitals) to the Cheshire and Merseyside Health Protection Unit (CMHPU) in England. Of these, 359 (27\%) were laboratory-confirmed and 522 (39\%) were discarded after laboratory results showed them to be PCR-negative.

\begin{abstract}
Background
Measles vaccination was introduced in the United Kingdom (UK) in 1968 with the combined measlesmumps-rubella (MMR) vaccine being used since 1988 [1]. As uptake of MMR vaccination increased, the numbers of confirmed cases of measles reduced. Confidence in the MMR vaccine has not always been good as controversies about potential links between MMR vaccine and autism in the late 1990s, which were later proved unfounded [2,3], show uptake in the UK reduced from $92 \%$ in 1996 to $80 \%$ in $2003[4,5]$. Outbreaks in recent years have mainly occurred in areas of the country or specific groups in which uptake of MMR vaccine is low [6-8].
\end{abstract}

Merseyside is an area in the north-west of England with a population of around 1.4 million people. Liverpool is

\section{FIGURE 1}

Number of confirmed and probable cases of measles by date of disease onset, Merseyside, England, January-June 2012 $(n=516)$

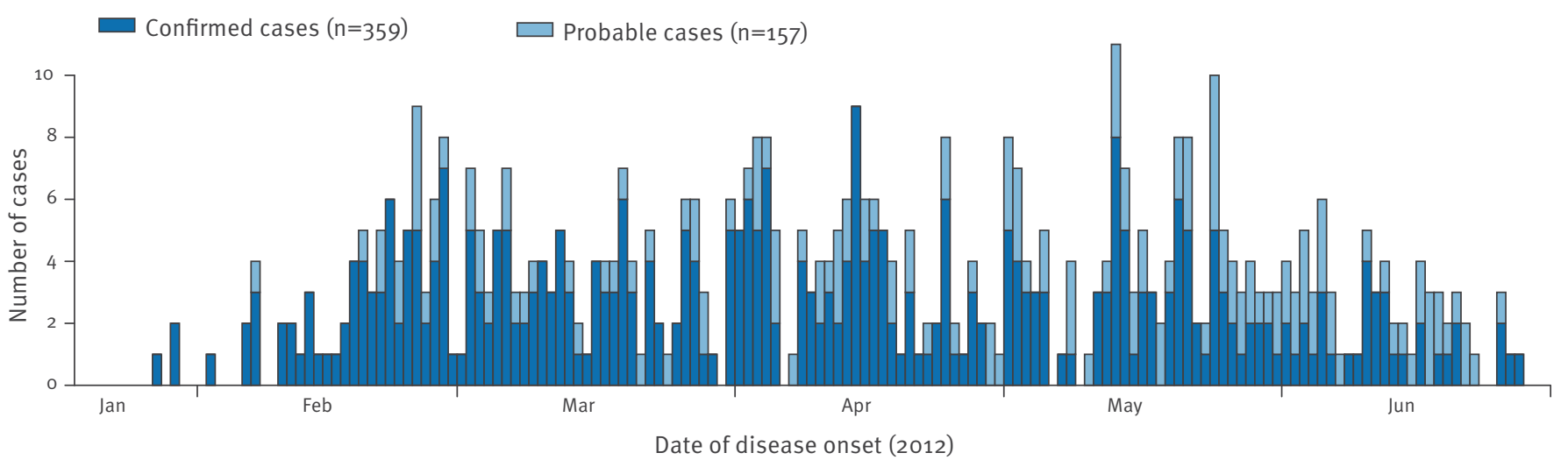


the largest metropolitan area in Merseyside. In recent years, uptake of the first dose of MMR vaccine (given from 13 months of age as part of the routine immunisation schedule in the UK) by 24 months of age has been over $92 \%$. Uptake by the age of five years of the second dose (normally offered from the age of three years and four months to five years) has been around $85 \%$. Despite recent small and localised outbreaks of measles in 2009 and 2011 in the neighbouring county of Cheshire, cases in Merseyside have been infrequent and sporadic.

\section{Outbreak description}

\section{Case definition}

A measles case definition was adapted from national guidelines to reflect local epidemiology [1]. A case was defined as probable when the three following criteria were fulfilled:

- Clinical presentation: fever and measles-like rash and one or more of the following symptoms: cough, conjunctivitis, coryza, or Koplik's spots.

- Residence / reported from: residence in Liverpool or adjacent areas, or being a close contact of a confirmed or probable case of measles, or history of recent travel to endemic or outbreak areas.

- MMR vaccination status: patients who had not received two doses of MMR vaccine (irrespective of age) or with unknown history of MMR vaccination. As most cases at the outset of the outbreak were unvaccinated and partially vaccinated, MMR vaccination status was considered in the case definition for practical purposes.

All reported cases not meeting the above criteria were considered as possible. All reported cases were sent a salivary self-testing kit by post or tested in healthcare settings. Cases were considered confirmed if they were measles IgM-positive on saliva, or if they had a positive PCR result from urine, saliva or throat swab. After the first confirmed case of measles in January 2012, all patients were also asked to respond to questions on age, sex, travel history, place of residence, vaccination status, and whether they had been in contact with vulnerable individuals.

\section{Epidemiology}

From 1 January until 30 June 2012, 1,339 suspected cases of measles were reported by clinicians (family doctors or hospitals) to CMHPU, of which 359 (27\%) were laboratory-confirmed and 522 (39\%) were discarded after laboratory results showed them to be PCR-negative. Of the remainder number of cases, based on the case definition, 157 (12\%) were classified as probable cases and the rest of 301 cases (22\%) were defined as possible. Although there is a statutory duty on doctors to report suspected cases of measles, the number of suspected cases that could have gone unreported is unknown. The epidemic curve shows a typical propagated outbreak pattern (Figure 1). Most cases

\section{FIGURE 2}

Measles-mumps-rubella vaccination status by age of (A) confirmed $(n=359)$ and $(B)$ probable $(n=157)$ measles cases, Merseyside, England, January-June 2012

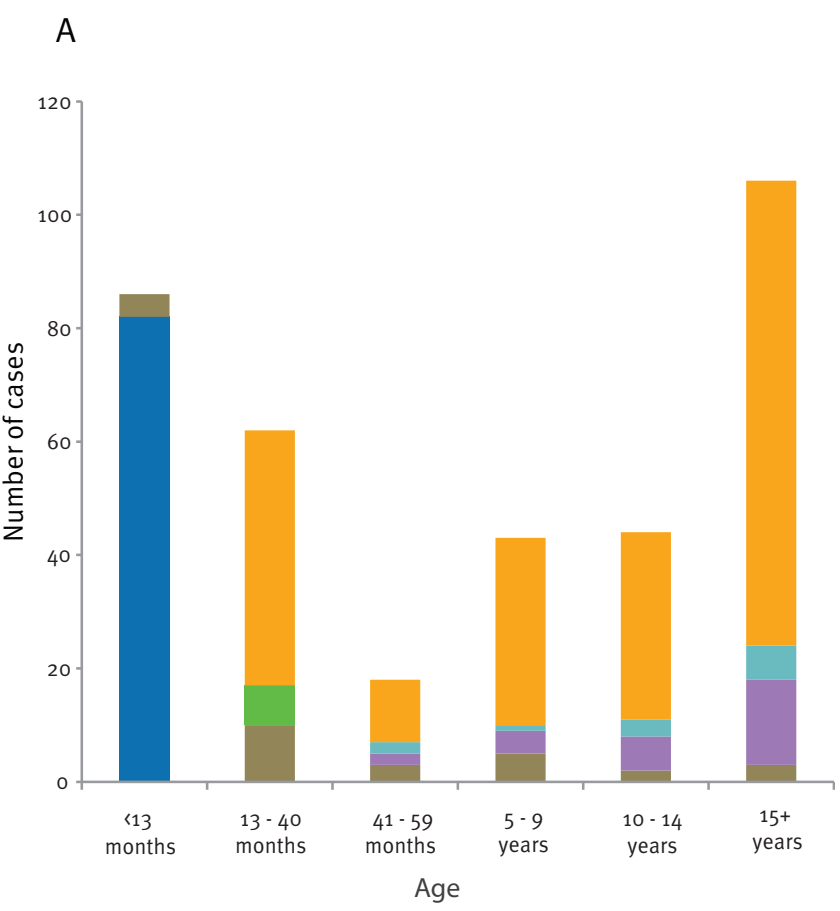

B

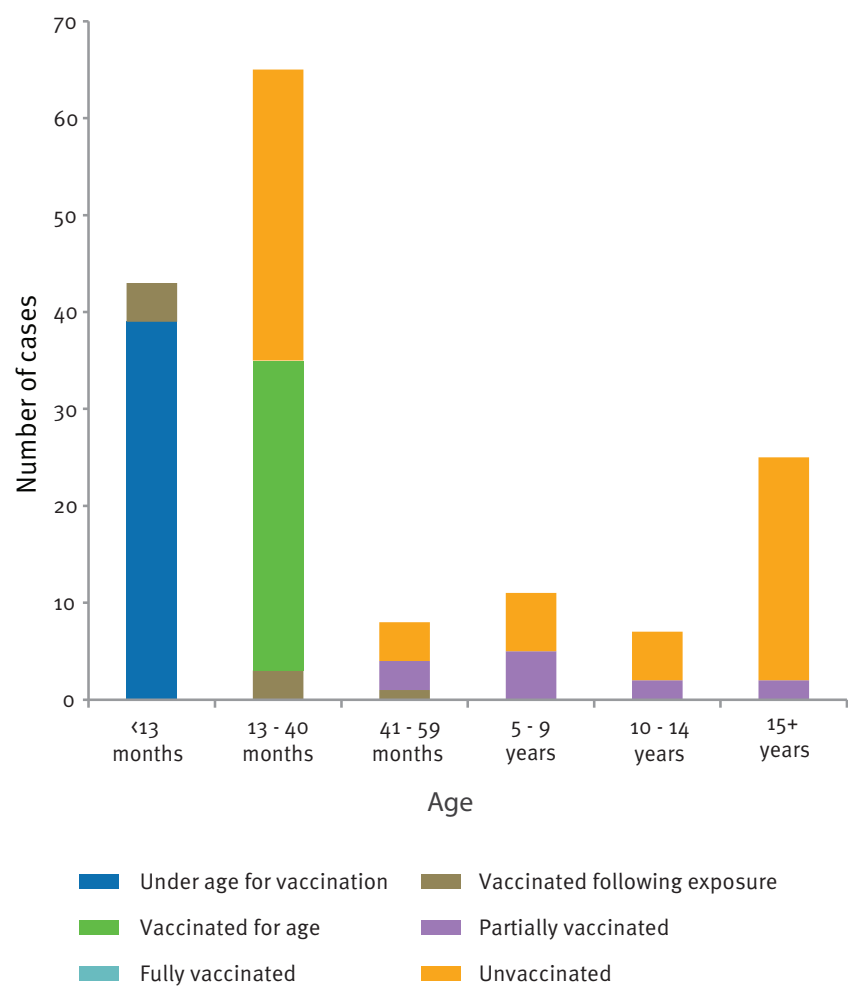


have been sporadic with very few outbreaks in nurseries. Some family clusters were also identified.

Of the 54 confirmed cases reported before March, at least 16 had documented exposure to another case in a healthcare setting (e.g. waiting rooms in clinics). To date, at least eight confirmed cases out of 359 (2\%) occurred in medical staff exposed to cases at work.

The reproductive number $\left(R_{0}\right)$, estimated through Poisson regression in week 3 after the start of the outbreak was $R_{0}=1.2$ (95\% Cl: 1.1-1.3), which slowly came down to an $\mathrm{R}_{0}=1.00(95 \% \mathrm{Cl}: 0.986-1.018)$ for confirmed cases in week 26.

Of the 359 confirmed cases, 175 (49\%) were female, 84 (23\%) were under one year of age and too young to be vaccinated and 106 (30\%) were 15-years-old or older. Of the cases aged less than 13 months, most were aged between 6 and 12 months $(n=68)$ and only two cases were under three months of age. Most confirmed cases eligible for vaccination, i.e. $>12$ months old, were not fully vaccinated $(38 \%$ had no previous MMR vaccination, $16 \%$ had only one dose of MMR) and information was not available for 68 (19\%) cases (Figure 2). Twelve of the confirmed cases had been vaccinated with two doses of MMR vaccine. There have been no measlesrelated deaths to date.

Overall, $63(18 \%)$ of the confirmed cases required hospitalisation, whereas only three $(2 \%)$ of the probable cases needed to be admitted to hospital. Hospitalisation rates in confirmed cases were higher in the very young ( $29 \%$ in children aged under one year) and older patients ( $41 \%$ of those aged 15 years and older). These figures are likely to underestimate true hospitalisation rates as admission to hospital after a case is reported is unlikely to be captured. Work is ongoing to accurately estimate the number of cases hospitalised during this outbreak.

Most confirmed cases were distributed in and around Liverpool and the neighbouring areas $(60 \%$ in Liverpool, $15 \%$ in Knowsley and $12 \%$ in Sefton), although confirmed cases of measles have been reported in all eight of the Primary Care Trusts (PCTs) in Cheshire and Merseyside (Figure 3).

\section{Laboratory results}

The genotype of the measles virus identified in 86 cases in Merseyside is B3. This is the predominant genotype circulating in Africa, although it has been reported in 2011 in outbreaks in other parts of Europe $[9,10]$. Genotype D8 has been identified in seven of the cases reported in Cheshire. D8 strain has also been identified in neighbouring areas: an ongoing outbreak in North Wales and sporadic cases in the Greater Manchester area. Both genotypes are distinct from that previously predominant in the UK (D4) [11]. Although the strain of measles is assumed to have been imported, it has not been possible to confirm this yet, as none of initial probable or confirmed cases

\section{FIGURE 3}

Geographical distribution of confirmed $(n=359)$ and probable $(n=157)$ measles cases, Merseyside, England, January-June 2012

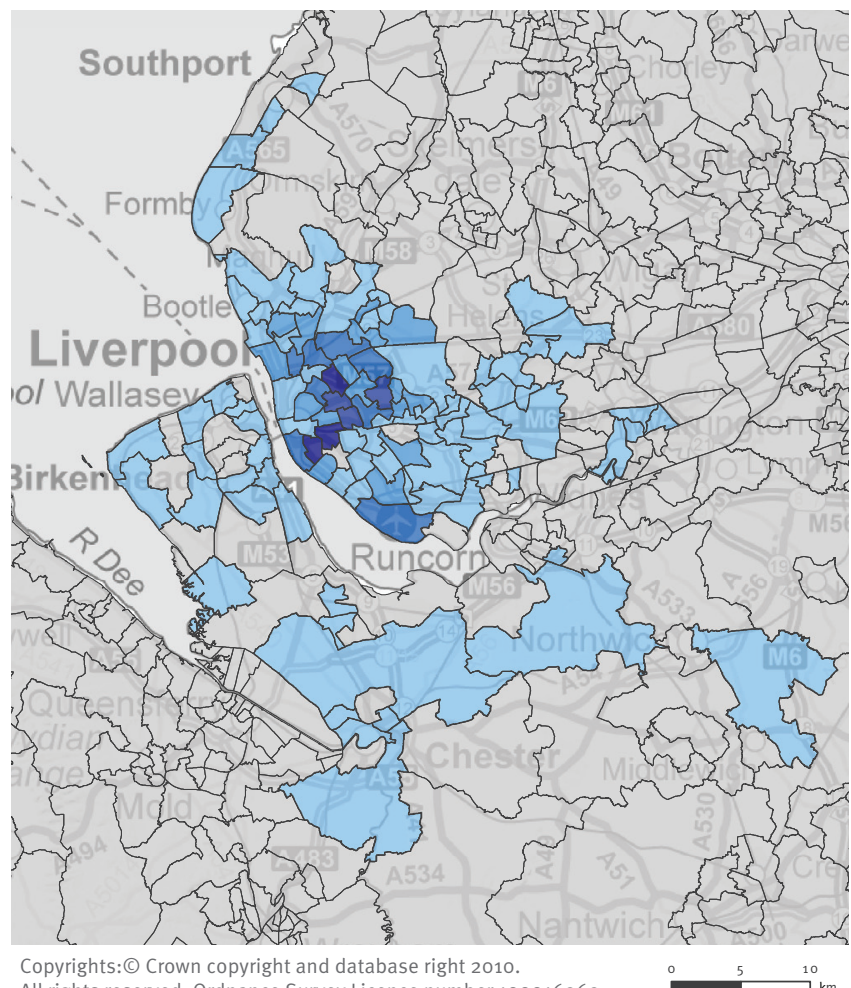

All rights reserved. Ordnance Survey Licence number 100016969

Number of cases

$$
\begin{aligned}
& 1-5 \\
& 6-10 \\
& 11-15 \\
& 16-20 \\
& >20
\end{aligned}
$$

reported since January in this outbreak had travelled abroad in the incubation period.

\section{Public health actions}

To minimise secondary spread and complications in vulnerable cases, individual risk assessment and contact tracing were conducted and, where appropriate, post-exposure prophylaxis (PEP) of vulnerable contacts (those under 13 months of age, pregnant women and immunocompromised persons) with either MMR vaccine or Human Normal Immunoglobulin (HNIG) was offered as per UK national guidelines [12]. Immunocompromised and pregnant contacts were offered HNIG if they were antibody-negative; infants from 0 to 5 months were offered HNIG, unless their mother was born before 1970 or if the mother had natural measles and was born between 1970 and 1984; infants from six months of age were offered MMR vaccination. Over 1,800 individual risk assessments have been conducted between January and June to ascertain vulnerable contacts and offer PEP. Over 1,000 contacts 
were followed up, and over 150 doses of HNIG have been issued to vulnerable contacts.

A Measles Response Centre was set up with support from the eight PCTs in Cheshire and Merseyside to manage the increased workload associated with contact tracing and risk assessment and a clinic was set to facilitate urgent administration of PEP.

\section{Increased vaccination efforts}

Existing initiatives to increase $M M R$ vaccination uptake in children and young people have been intensified by primary care and community healthcare staff. Families of children not fully vaccinated have been contacted to encourage vaccination during March and May. Effort has been made to make it as easy as possible for people to take up the opportunity to be vaccinated, e.g. through setting up community clinics at convenient times or home visits. Vaccination was offered at the small number of schools and nurseries where clusters of cases were reported. Coverage statistics for Liverpool, where the majority of the cases occurred, showed uptake improvements from the last quarter of 2011 (October to December) to the first quarter of 2012 (January to March): uptake for the first dose of MMR vaccine by two years of age improved from $95.1 \%$ to $95.5 \%$, and for the second dose by five years of age from $85.6 \%$ to $88 \%$ [13]. Provisional figures for the quarter from April to June 2012 suggest further improvements to $96 \%$ and $90 \%$ respectively. Similar improvements have been seen in other areas in Merseyside. Additionally, in March alone, over 1,500 children and young adults (aged from 5 to 24 years) were vaccinated across Merseyside. Quarterly coverage data for the period April to June 2012 are not yet available.

\section{Awareness campaign}

An intensive media campaign has been mounted with regular press releases issued by the Health Protection Agency on behalf of the Multiagency Outbreak Control Team informing about the outbreak, persons at risk of getting the infection, what to do if infected to minimise exposure to others, and ongoing public health measures. This received coverage by most local and some national newspapers, radio and television on repeated occasions [14].

\section{Discussion and conclusions}

This measles outbreak is by far the largest in the North West of England at least since 1996 [15]. Around a quarter of cases have, so far occurred in children too young to be vaccinated. This age group is normally dependant on passive immunity (maternal antibodies) or herd immunity for protection. Given that the uptake of the first dose of MMR vaccine locally in recent years has been relatively high, this large number of cases can partially be explained by the lower levels of immunity in older children and teenagers, and by lower levels of maternal antibodies passed on to babies from vaccinated mothers [16]. Therefore a priority in the control of community outbreaks like this should be to target older children and young adults who missed vaccination in childhood. Lowering the age of the first MMR as a control measure was discussed by the outbreak control team but it was discarded as it was thought that it could divert attention and resources from improving uptake of the routine vaccination programme an the catch up of older children and younger adults.

The low proportion of cases seen in infants less than five months of age is likely to be the result of passive immunity, which wanes later in the infant period [16], hence the larger proportion of cases in children aged between 6 and 12 months.

Exposure to measles in healthcare settings is described in the literature as a risk for acquiring measles and propagating outbreaks $[17,18]$. In the outbreak described here, a number of measles exposures occurred in healthcare settings due to the difficulty in clinically differentiating measles, particularly in its early stages, from other viral systemic illnesses and the challenge of maintaining airborne precautions in waiting rooms. After isolation of suspected cases and cohorting were put in place in emergency rooms and community clinics, exposures and secondary transmission have been reduced.

Interestingly, the genotype (B3) of measles virus seen in this outbreak is distinct from that of outbreaks seen in the UK in 2010 and 2011. Circulation of B3 in the UK was uncommon before this outbreak, but it has caused outbreaks in Europe in the last three years [10]. The genotype is assumed to have been imported but it has not been possible to determine how it was introduced in Merseyside.

Acknowledgments

We would like to acknowledge the hard work put in the management and control of this outbreak by the team at the CMHPU and all the individuals, departments and organisations that have been involved since the start of the outbreak: all general practitioners in Merseyside; Aintree University Hospital; Alder Hey Children's Hospital; Liverpool Community Health; Liverpool Women's Hospital; Royal Liverpool \& Broadgreen University Hospital; St Helens \& Knowsley NHS Trust; Halton \& St Helens Primary Care Trust; Knowsley Primary Care Trust; Liverpool Primary Care Trust; Sefton Primary Care Trust; Wirral Primary Care Trust; North West Public Health Trainees; Cumbria \& Lancashire HPU; Greater Manchester HPU; HPA Immunisation Division (Colindale); HPA North West Laboratory (Manchester); and HPA North West Regional Office and Epidemiology Unit. 


\section{References}

1. Health Protection Agency (HPA). HPA National Measles Guidelines Local \& Regional Services. Version 1.2. London: HPA. 28 Oct 2010. Available from: http://www.hpa.org.uk/ webc/HPAwebFile/HPAweb_C/1274088429847

2. Taylor B, Miller E, Farrington CP, Petropoulos MC, FavotMayaud I, Li J, et al. Autism and measles, mumps, and rubella vaccine: no epidemiological evidence for a causal association Lancet. 1999;353(9169):2026-9.

3. Farrington P, Miller E, Taylor B. MMR and autism: further evidence against a causal association. Vaccine. 2001;19(27):3632-5.

4. Health Protection Agency (HPA). Completed primary courses at two years of age: England and Wales, 1966-1977, England only 1978 onwards. London: HPA. 21 Oct 2011. Available from: http://www.hpa.org.uk/web/HPAweb\&HPAwebStandard/ HPAweb_C/1195733819251

5. Choi YH, Gay N, Fraser G, Ramsay M. The potential for measles transmission in England. BMC Public Health. 2008;8:338.

6. Health Protection Agency (HPA). Confirmed measles, mumps and rubella cases in 2007: England and Wales. Health Protection Report. 2008;2(8). 22 Jan 2008. Available from: http://www.hpa.org.uk/hpr/archives/2008/news0808.htm

7. Health Protection Agency (HPA). Confirmed measles cases in England and Wales, January 2008 to February 2009. Health Protection Report. 2009;3(13). 3 Apr 2009. Available from: http://www.hpa.org.uk/hpr/archives/2009/news1309.htm

8. Health Protection Agency (HPA). Confirmed measles cases in England and Wales - update to end-January 2011. Health Protection Report. 2011;5(8). 25 Jan 2011. Available from: http://www.hpa.org.uk/hpr/archives/2011/news0811.htm

9. World Health Organization (WHO). Measles outbreaks in Europe. Wkly Epidemiol Rec. 2011;86(18):173-4.

10. World Health Organization (WHO). Measles surveillance data. Immunization surveillance, assessment and monitoring. Geneva: WHO. 28 Jun 2012. Available from: http://www.who. int/immunization_monitoring/diseases/measles_monthlydata/ en/index1.html

11. Health Protection Agency (HPA). Laboratory confirmed cases of measles, mumps and rubella in England and Wales: January to March 2012. Health Protection Report. 2012;6(21). 25 May 2012. Available from: http://www.hpa.org.uk/hpr/ archives/2012/hpr2112.pdf

12. Health Protection Agency (HPA). Post exposure prophylaxis for measles: Revised guidelines. London: HPA. May 2009. Available from: www.hpa.org.uk/web/HPAwebFile/ HPAweb_C/1238565307587

13. Health Protection Agency (HPA). Quarterly vaccine coverage data tables. London: HPA. [Accessed: 17 Jul 2012]. Available from: http://www.hpa.org.uk/web/HPAweb\&HPAwebStandard/ HPAweb_C/1211441442288

14. Health Protection Agency (HPA). North West Press Releases London: HPA. [Accessed 17 Jul 2012]. Available from: http:// www.hpa.org.uk/web/HPAweb\&Page\&HPAwebAutoListNameD esc/Page/1274089139877

15. Health Protection Agency (HPA). Confirmed cases of measles by region and age: 1996-2011. London: HPA. 17 Feb 2012. Available from: http://www.hpa.org.uk/web/ HPAweb\&HPAwebStandard/HPAweb_C/1195733778332

16. Leuridan E, Hens N, Hutse V, leven M, Aerts M, Van Damme P. Early waning of maternal measles antibodies in era of measles elimination: longitudinal study. BMJ. 2010;340:C1626.

17. Farizo KM, Stehr-Green PA, Simpson DM, Markowitz LE. Pediatric Emergency Room Visits: A Risk Factor for Acquiring Measles. Pediatrics. 1991;87(1):74-9.

18. Chen SY, Anderson S, Kutty PK, Lugo F, McDonald M, Rota PA, et al. Health care-associated measles outbreak in the United States after an importation: challenges and economic impact. Infect Dis. 2011;203(11):1517-25. 